
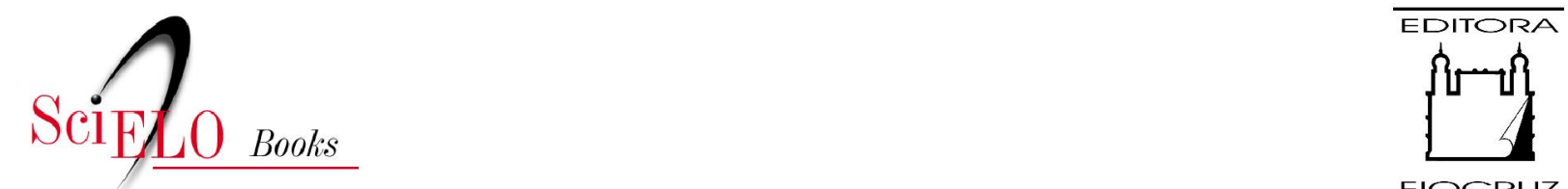

FIOCRUZ

\title{
Diseño e implementación de proyectos interculturales en la formación docente
}

\author{
María Isabel Pozzo
}

\section{SciELO Books / SciELO Livros / SciELO Libros}

POZZO, M. I. Diseño e implementación de proyectos interculturales en la formación docente. In: SOUZA, F. M., and ARANHA, S. D. G., orgs. Interculturalidade, linguagens e formação de professores [online]. Campina Grande: EDUEPB, 2016, pp. 231-250. Ensino e aprendizagem collection, vol. 2. ISBN 978-85-7879-347-0. Available from: doi: $10.7476 / 9788578793470.0011$. Also available in ePUB from: http://books.scielo.org/id/qbsd6/epub/souza-9788578793470.epub.

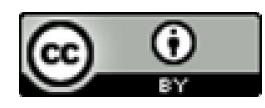

All the contents of this work, except where otherwise noted, is licensed under a Creative Commons Attribution $\underline{4.0 \text { International license. }}$

Todo o conteúdo deste trabalho, exceto quando houver ressalva, é publicado sob a licença Creative Commons Atribição 4.0.

Todo el contenido de esta obra, excepto donde se indique lo contrario, está bajo licencia de la licencia Creative Commons Reconocimento 4.0 . 


\title{
DISEÑO E IMPLEMENTACIÓN DE PROYECTOS INTERCULTURALES EN LA FORMACIÓN DOCENTE
}

\author{
María Isabel Pozzo (UNR)
}

En la República Argentina, la formación docente se desarrolla mayormente -aunque no exclusivamente- en los Institutos de Formación Docente (IFD), los cuales se ubican en el subsistema de educación superior no universitaria. Tradicionalmente, constituyeron un ámbito relegado del sistema educativo, ofuscado por las problemáticas imperiosas del ciclo educativo obligatorio y por el reconocimiento social del que goza el nivel universitario. La creación del Instituto Nacional de Formación Docente (INFD) en el marco de la vigente Ley de Educación Nacional, del 2006, vino a revertir este relegamiento, generando un ámbito específico en el cual prestar la imprescindible atención que merece la formación docente. En el nivel regional de la provincia de Santa $\mathrm{Fe}$, una de las zonas geográficas más pobladas y económicamente pujantes del país austral, la Secretaría de Ciencia, Tecnología e Innovación de la provincia de Santa Fe (hoy devenida 
en Ministerio) inauguró en 2013 el Programa de fortalecimiento de las capacidades del sistema de investigación y desarrollo en la provincia de Santa Fe para proyectos de investigación. Específicamente, creó un Instrumento de Apoyo y estímulo a Proyectos de investigación entre los Institutos de Educación Superior de la provincia y el Sistema científico radicado en el territorio provincial. Se trataba de una convocatoria nueva, tendiente a promover el desarrollo de la investigación en dichos institutos en virtud del trabajo conjunto con instituciones dedicadas a la investigación (como el Instituto Nacional de Tecnología Agropecuaria (INTA), el Instituto Nacional de Tecnología Industrial (INTI), o el Concejo Nacional de Investigaciones Científicas y Técnicas (CONICET).

En dicho marco, un equipo argentino coordinado por quien suscribe, presentó una propuesta a dicha convocatoria en una línea de trabajo que venía realizando desde hace tiempo, entre instituciones que tenían que ver, precisamente, con el perfil estipulado, pero que podían verse fortalecidos con el apoyo de un ente provincial de esta talla. En tanto la propuesta resultó seleccionada, se concretó un cluster interinstitucional compuesto por el Instituto Rosario de Investigaciones en Ciencias de la Educación (IRICE) del CONICET, el Instituto de Educación Superior (IES) - No 28 "Olga Cossettini" de Rosario. Además de las instituciones participantes acorde a la convocatoria, están presentes otras del subsistema universitario, tanto argentino como extranjero: el Centro de Estudios del Español como Lengua Extranjera y la Escuela de Ciencias de la Educación de la Universidad Nacional de Rosario, Argentina, la Universidad de Aarhus, Dinamarca, y la Universidad de Salerno, Italia. 
La convocatoria estipulaba desarrollar proyectos de investigación orientados a temas de interés provincial, los cuales se plasmaban en un listado establecido por la Secretaría de Ciencia, Tecnología e Innovación y el Ministerio de Educación al momento de la convocatoria. De todos ellos, el eje $\mathrm{n}^{\circ} 10$ : "Migraciones, pueblos originarios, multiculturalidad", se relacionaba con la línea de investigación del Área de estudios interculturales a mi cargo en el Instituto Rosario de Investigaciones en Ciencias de la Educación (IRICE-CONICET), así como los estudios que veníamos desarrollando en el Centro de Estudios del Español como Lengua Extranjera de la Facultad de Humanidades y Artes de la Universidad Nacional de Rosario.

En base a dichas convergencias temáticas, conformamos un grupo de profesores, graduados y estudiantes con quienes, en su mayoría, ya habíamos compartido proyectos previos y/o nos encontrábamos trabajando en conjunto en carácter de tesistas, becarios, alumnos o colegas. Constituimos así un equipo interdisciplinario formado en distintas disciplinas (Ciencias de la Educación, Historia, Letras, Inglés, Comunicación Social, Psicopedagogía). Otro rasgo a destacar es que, si bien casi todas somos argentinas, algunas residen desde hace mucho tiempo en el extranjero, situación que nos facilitó el trabajo con instituciones de otros países.

El antecedente más directo (por la composición del equipo, el perfil institucional y su temática) fue el proyecto “Una propuesta de desarrollo disciplinar e intercultural en la formación y perfeccionamiento docentes a partir de la alfabetización de migrantes en la ciudad de Rosario". Esta propuesta fue seleccionada y subsidiada por el Instituto 
Nacional de Formación Docente (INFD) en la convocatoria 2007 de proyectos concursables de investigación pedagógica "Conocer para incidir sobre los aprendizajes escolares". También en dicha oportunidad se trataba de una nueva estrategia institucional tendiente a promover la investigación en los Institutos de Formación Docente - aunque en dicha oportunidad, promovida por la esfera nacional -. El trabajo resultante arrojó un libro de autoría colectiva publicado en Alemania (POZZO, ed., 2009), además de ponencias y artículos. Como hicimos en dicha oportunidad, el proyecto relatado en este capítulo fue plasmado en una obra grupal (POZZO, ed., 2015), en la que los distintos capítulos reflejan distintos tramos del trabajo realizado desde el año 2014. El libro contó con el referato a cargo de un prestigioso comité académico conformado por la Dra Elena Achilli, profesora de la Universidad Nacional de Rosario, la Dra. Melina Porto (Universidad Nacional de La Plata y CONICET) y el Dr. José María Izquierdo (Federación Internacional de Asociaciones de Profesores de Español -FIAPE- y Universidad de Oslo, Noruega). A su vez, el libro está prologado por el Dr. Maximiliano Ritacco Real, un argentino originario de Bahía Blanca que se desempeña como profesor en el Departamento de Ciencias de la Educación de la Universidad de Zaragoza. Este encuadre institucional más el propio proyecto están descriptos en el primer capítulo (POZZO, 2015).

Además de dicho libro, plasmamos nuevos resultados en posteriores ponencias y artículos, por lo que este capítulo configura una puesta al día de nuestro trabajo. Asumimos las actividades de difusión con el convencimiento de que todo conocimiento producido recién finaliza su proceso cuando se socializa. 


\section{Proyectos interculturales en la formación docente}

Examinar la temática de la interculturalidad en la formación docente admite diversas puertas de ingreso. Una muy fecunda es la reflexión sobre la categoría de competencia comunicativa intercultural (CCI), propuesta originariamente por Byram (1997) y adoptada en un documento tan influyente como en Marco Comúne Europeo de Referencias para las lenguas (CONSEJO DE EUROPA, 2002). Su definición requiere remontarse a un examen de la noción de competencias, llevando luego la reflexión a la particularidad de la CCI (POZZO; PIDELLO, 2015). Nos proponemos cubrir la vacancia que existe en torno a la figura del profesor, dado el importante rol que les cabe como responsables de promover dicho desarrollo y por el especial interés que reviste en su reposicionamiento como transmisor cultural.

Otros abordajes vinculan el concepto de competencia comunicativa intercultural con el de comunicación didáctica (PAROLA, 2015). Al tratarse este último de un contenido habitualmente frecuente en los Programas de las asignaturas pedagógicas, es factible vincular la reflexión teórica desde diversas materias (por ejemplo: Ateneo de Educación Superior con Teoría del Curriculum) e incluso profesorados. A su vez, considerando la composición multicultural de la sociedad actual es posible encararlo con una experiencia áulica a partir de la presencia de estudiantes extranjeros. Los Profesorados de Lenguas Extranjeras suelen contar además con asistentes de lengua. Temas relativos a la interculturalidad y formación docente son también motivo de cursos de capacitación extracurriculares, los cuales resultan muy propicios para 
relevar concepciones de los docentes acerca de qué es la interculturalidad (FERNÁNDEZ; DAVANTURE, 2015).

Partir de situaciones de distancia cultural en escenarios naturales (no experimentales) de la formación docente requiere una fase de diagnóstico tendiente a describir el contexto institucional en cuanto a la presencia de migrantes y visitantes en la educación superior. Al respecto cabe tener presente la relevancia de la movilidad académica estudiantil como objeto de estudio. Vale decir, las oportunidades existentes en cuanto a convocatorias, pero también cómo estas han sido motivo de investigación en cuanto al logro de sus objetivos, sus continuidades y rupturas, etc (VIRGOLÍN; POZZO, 2015). En tal sentido, la peculiaridad de la educación superior no universitaria en Argentina mencionada previamente requiere un examen específico de los programas de movilidad académica disponibles para la formación docente (BERTRAMO; D'ASCANIO; POZZO, 2015). Nuestro cotejo dejó ver la menor disponibilidad de oportunidades para los institutos terciarios, a cargo principalmente de las instituciones de lenguas extranjeras (Alianza Francesa o Concejo Británico) y, de manera reciente, por el INFD. Tras este encuadre general sobre el estado de situación de la internacionalización de la educación superior en Argentina, nos abocamos a nuestro caso en estudio, el Instituto de Educación Superior $\mathrm{N}^{\circ} 28$ "Olga Cossettini" de Rosario, Argentina. Allí realizamos un estudio situado de la presencia de extranjeros por dos medios: a través de un exhaustivo relevamiento a través del Sistema de Gestión Escolar Web (SIGAE) del Ministerio de Educación de la Provincia de Santa Fe (AMEZ, 2015), y por medio de entrevistas abiertas para conocer las historias de los estudiantes extranjeros en el Instituto desde su 
propia percepción (BERTRAMO; D'ASCANIO; POZZO, 2015). Y considerando que nuestra población objeto son futuros docentes de escuela media, relevamos el perfil de los estudiantes de intercambio, que constituyen el grupo más numeroso de extranjeros en las escuelas secundarias argentinas (JACOB; POZZO, 2015a).

Una vez realizados los estudios diagnósticos a escala nacional, regional e institucional en torno al caso en estudio, es posible iniciar la fase de intervención. Partimos de la unidad de análisis que hemos dado en llamar "espacios interculturales" -tomando la expresión de DÍAZ; ALONSO (2004) a partir de la presencia de población extranjera en la institución según se desprende del relevamiento de la fase anterior y fuera de ella por medio de las TICs. Dichos espacios se tornan en proyectos una vez que alcanzan una acción planificada al interior del grupo-clase, sea en una o varias asignaturas y carreras en la formación docente. Para diseñar e implementar nuestros proyectos interculturales, seleccionamos carreras humanísticas de la formación inicial que se dictan en el Instituto de Formación Docente seleccionado: los Profesorados de Francés lengua Extranjera e Historia, cuya formación didáctica examinamos (D'ASCANIO; BERTRAMO; POZZO, 2015). Dentro de estas carreras identificamos espacios curriculares cuyos contenidos pueden articularse a un proyecto intercultural. Asimismo, resolvimos incluir el área artística, para lo cual fue necesario recurrir a otra institución de formación docente en la cual esta área integra el curriculum. Así, involucramos a los Profesorados de Nivel Inicial y Primaria de la Escuela Provincial No 35 (ex Normal No 2 ) de Rosario. 
En dichos marcos, diseñamos e implementamos proyectos interculturales valiéndonos de dos instancias:

1) la presencia de estudiantes extranjeros en situación de movilidad (migrantes y estudiantes de intercambio);

2) las posibilidades de intercambio con población extranjera que proporcionan las TICs.

En el primer caso, implementamos un taller de reflexión en torno a distintos tópicos asociados al tema de la interculturalidad (PAROLA, 2015). El espacio elegido fue el de Teoría del Curriculum en el Profesorado de Francés, que es el que contiene la mayor cantidad de población extranjera, principalmente haitianos (según se advierte en AMEZ, 2015a). La reflexión dio lugar a una investigación de campo en el que los docentes en formación diseñaron un protocolo de encuesta y lo aplicaron a profesores y estudiantes nativos y extranjeros.

En la clase de educación artística en la formación de maestros, los estudiantes identificaron distintas producciones musicales de la ciudad, analizando los marcos de recepción de las mismas (POLO, 2015). Tanto los estudiantes del profesorado de francés como los de magisterio plasmaron los resultados obtenidos en producciones audiovisuales disponibles en el sitio web del proyecto ${ }^{1}$.

Con los alumnos del Profesorado de Historia diseñamos un proyecto intercultural mediado por las TICs en conjunto con cursos de español de universidades extranjeras no hispanohablantes cuyas profesoras pertenecen a

1 https://sites.google.com/site/espaciosinterculturales/ el-proyecto. 
nuestro equipo de investigación. Una de ellas es Susana Fernández (de la Universidad de Aarhus, Dinamarca), para cuyos alumnos se confeccionó el texto histórico que sustentó el intercambio virtual (GARCÍA; POZZO, 2015a). La otra profesora es Margarita Theiner (de la Universidad de Salerno, Italia), con cuyos alumnos estructuramos el intercambio en torno a entrevistas sobre temáticas locales (THEINER; POZZO, 2015). Estos proyectos inauguran el eje de cultura regional de nuestro proyecto, ya que refiere a la provincia de Santa $\mathrm{Fe}$, en la cual está ubicada nuestra ciudad de Rosario, en tiempos de la colonia español. Vale decir que acercamos a estudiantes europeos de español a cuestiones de nuestra provincia, que a la luz de Hispanoamérica resulta un punto pequeño, y sin embargo, digno de atención. Además del contenido que estructura el intercambio, realizamos una reflexión sobre la experiencia de telecolaboración (FERNÁNDEZ; POZZO, 2015) para considerar en futuras réplicas.

A partir del interés que suscitaron estas iniciativas entre nuestros contactos internacionales, implementamos un proyecto similar en esta modalidad con una escuela secundaria de Francia (BÉRODOT; POZZO, 2015). La diferencia de edad entre los participantes marcó una situación homologable a la que tendrán los futuros docentes de Historia, a lo que se suma la ventaja lingüística de los mayores al ser hablantes nativos de la lengua del intercambio virtual. También llevamos a cabo un proyecto de comunicación asincrónica con alumnos de Medicina de una universidad brasileña -UNILA, de Foz de Iguazú. La especificidad disciplinar de la carrera brasileña participante fue atendida desde lo histórico. Así, los estudiantes argentinos de Historia y su profesora confeccionaron un 
texto para el intercambio (GARCÍA; POZZO, 2015b), también centrado en la provincia de Santa Fe, y filmaron su relato oral. Una vez subido a internet, este pudo ser visitado por los estudiantes brasileños de medicina.

Para estos proyectos que se valieron de las posibilidades que ofrecen las TICs, realizamos un diagnóstico de las habilidades tecnológicas de los estudiantes del profesorado de Historia (JACOB; POZZO, 2015b). Si bien se trata de un grupo joven (promedian los 22 años), dieron prueba del desconocimiento de algunas herramientas tecnológicas básicas para la implementación de proyectos mediados por las TICs (tales como skype o power point), lo cual nos implicó dedicar un tiempo previo a su familiarización.

Del Profesorado de Historia elegimos el Seminario de Historia regional por dos razones: por el carácter aglutinante de este campo de estudio que involucra tanto contenidos disciplinares como elementos identitarios, y en tanto la historia latinoamericana es parte de la currícula de las carreras de español como lengua extranjera. En las sesiones de telecolaboración con las instituciones europeas, los profesores en formación debían transmitir la propia cultura a estudiantes extranjeros según un plan cuidadosamente estipulado.

Todos estos proyectos fueron evaluados por sus protagonistas, así como por los docentes-investigadores a cargo según un sistema operacional de indicadores de la CCI. Los resultados obtenidos fueron socializados en diversos eventos y publicaciones académicas (POZZO; 2015b; POZZO et al., 2015). Las producciones contribuyen a superar la vacancia señalada por YOUNG y SACHDEV (2011, p.81), quienes resaltan los escasos estudios empíricos sobre cómo se llevan a la práctica los enfoques 
interculturales, relegados por estudios teóricos o de naturaleza retórica.

En una fase de aplicación, y en vistas a dotar a los resultados de un impacto en el plano didáctico, construimos materiales didácticos en formato audiovisual para la enseñanza de historia argentina y latinoamericana. Esto es, en esta instancia ya capitalizamos las experiencias previas pero nos focalizamos más en el producto que en el proceso. La propuesta de hacer audiovisuales de historia latinoamericana y argentina responde a la vacancia que detectamos en este soporte, así como la escasa presencia de Latinoamérica como contenido de los libros de español como lengua extranjera que relevamos en estudios previos (POZZO, 2014). Al mismo tiempo, percibíamos al interés que esta región del planeta está teniendo en diversas universidades de países no hispanohablantes. Las lecciones en videos están concebidas para estudiantes extranjeros, en cuanto a la explicitación de referentes culturales, pero resultan aplicables también a población nativa por su claridad explicativa. Estos cubren los siguientes recortes espacio-temporales y temáticos:

- El contexto internacional, siglo XIX: “El largo y tumultuoso siglo XIX";

- América Latina, siglo XIX: "América o las Américas";

- América Latina, siglo XX: "América Latina en el siglo XX. Revoluciones y contra-revoluciones";

- Argentina 1880-1912: “La consolidación del Estado nacional argentino". 
Todos los videos se encuentran disponibles gratuitamente en el sitio web del proyecto ya indicado. La selección de contenidos para su tratamiento comprimido en una duración no mayor a quince minutos es el principal desafío para los guionistas. Por ellos, los criterios históricos y educativos de la realización audiovisual ameritan una producción propia tendiente a explicitar los fundamentos. Así, los tres primeros videos, abocados cada uno de ellos a cien años de historia, nada menos, se encuentran desarrollados en: POZZO; BESSO PIANETTO; DONATO, 2015; BESSO PIANETTO; POZZO, 2014; POZZO; BESSO PIANETTO, 2016, respectivamente. Por su parte, el aprovechamiento didáctico probado con estudiantes italianos puede ser consultado en THEINER, 2015. Las actividades basadas en el recurso audiovisual pueden ser transferidas a una plataforma académico-institucional, tarea que plantea cuestiones teóricas y metodológicas al docente (AMEZ, 2015b) atendiendo a las peculiaridades contextuales.

La atención a los aspectos metodológicos inherentes al diseño de proyectos (FANDINO CUBILLOS, 2007) permite contribuir a nuevas implementaciones tanto propias como ajenas. Por otra parte, optar por proyectos interculturales es una posibilidad para todos aquellos docentes que deseen innovar sus prácticas apelando a la naturaleza misma de la sociedad actual. Es con dicha intención que este capítulo se integra al presente libro, como una invitación constantemente abierta a sus lectores y con un presente de vínculos interinstitucionales encaminados a robustecer la formación docente en el mundo. 


\section{REFERENCIAS}

AMEZ, Mariel. La presencia de estudiantes extranjeros en el nivel superior argentino. Un estudio de caso centrado en el Instituto de Educación Superior $N^{\circ} 28$ "Olga Cossettini" de Rosario. En POZZO, María Isabel (ed.) Construcción de espacios interculturales en la formación docente: competencia comunicativa intercultural, cultura regional y TIC, p.71-84. Rosario: Laborde Editor. 2015a. ISBN 978-987-677-109-2.

AMEZ, Mariel. Construcción de materiales didácticos mediados por TIC: consideraciones teóricas y decisiones preliminares. En POZZO, María Isabel (ed.) Construcción de espacios interculturales en la formación docente: competencia comunicativa intercultural, cultura regional y TIC, p.245-254. Rosario: Laborde Editor. 2015b. ISBN 978-987-677-109-2.

BÉRODOT, Solène; POZZO, María Isabel. Las TIC en la clase de lengua extranjera. Un proyecto de coacción entre docentes argentinos en formación y estudiantes franceses de escuela media. En POZZO, María Isabel (ed.) Construcción de espacios interculturales en la formación docente: competencia comunicativa intercultural, cultura regional y TIC, p.191-202. 2015. Rosario: Laborde Editor. ISBN 978-987-677-109-2.

BESSO PIANETTO, María Elena; POZZO, María Isabel. Reflexiones teórico-metodológicas para la realización de un audiovisual educativo sobre América Latina. Revista Anuario Americanista Europeo, n.12, p.71-86. ISSN 22213872. Consejo Europeo de Investigaciones Sociales de 
América Latina. Disponible en: http://www.red-redial. net/revista/anuario-americanista-europeo/article/viewFile/278/276. 2014.

BYRAM, Michael. Teaching and assesing intercultural communicative competence. Clevedon: Multilingual Matters, 1997.

CONSEJO DE EUROPA (2001). Marco Común Europeo de Referencia para las lenguas: aprendizaje, enseñanza, evaluación. Traducido al español por el Instituto Cervantes. Madrid: Anaya, 2001.

DÍAZ, Rubén; ALONSO, Graciela. Construcción de espacios interculturales. Buenos Aires: Miño y Dávila, 2004.

FANDIÑO CUBILLOS, Graciela. El pensamiento del profesor sobre la planificación en el trabajo por proyectos. Bogotá: Universidad Pedagógica Nacional, 2007.

FERNÁNDEZ, Marcela; DAVANTURE, Adriana. La contextualización como eje del enfoque intercutural-multicultural. Experiencias de profesores de francés. En POZZO, María Isabel (ed.) Construcción de espacios interculturales en la formación docente: competencia comunicativa intercultural, cultura regional y TIC, p.121-130. Rosario: Laborde Editor, 2015. ISBN 978-987-677-109-2.

FERNÁNDEZ, Susana Silvia; POZZO, María Isabel. La telecolaboración como herramienta para la enseñanza/ aprendizaje de la historia regional argentina en Dinamarca: un proyecto de intervención didáctica. En POZZO María Isabel (ed.) Construcción de espacios interculturales 
en la formación docente: competencia comunicativa intercultural, cultura regional y TIC, p.155-180. Rosario: Laborde Editor, 2015. ISBN 978-987-677-109-2.

GARCÍA, Lía; POZZO, María Isabel. Santa Fe: territorio de los primeros enclaves coloniales tras la conquista. En POZZO, María Isabel (ed.) Construcción de espacios interculturales en la formación docente: competencia comunicativa intercultural, cultura regional y TIC, p.181-190. Rosario: Laborde Editor, 2015a. ISBN 978-987-677-109-2.

GARCÍA, Lía Claudia; POZZO, María Isabel. Salud y enfermedad en la ciudad de Rosario. Un abordaje histórico para estudiantes brasileños de español de la carrera de Medicina. En POZZO, María Isabel (ed.) Construcción de espacios interculturales en la formación docente: competencia comunicativa intercultural, cultura regional y TIC, p.203-220. Rosario: Laborde Editor, 2015b. ISBN 978-987-677-109-2.

PAROLA, Gilda. La formación inicial docente: momento clave para el desarrollo de la competencia comunicativa intercultural. En POZZO, María Isabel (ed.) Construcción de espacios interculturales en la formación docente: competencia comunicativa intercultural, cultura regional y TIC, p.99-120. Rosario: Laborde Editor, 2015. ISBN 978-987-677-109-2.

POLO, María del Pilar. Postales musicales de la ciudad de Rosario: un aporte desde el Área Estético-Expresiva a la construcción de competencias comunicativas interculturales en la Formación Docente. En POZZO, María Isabel (ed.) Construcción de espacios interculturales en la 
formación docente: competencia comunicativa intercultural, cultura regional y TIC, p.273-284. Rosario: Laborde Editor, 2015. ISBN 978-987-677-109-2.

POZZO, María Isabel (Ed.). Migraciones y formación docente. Aportes para una educación intercultural. Berlín: Peter Lang, 2009. ISBN: 978-3-0343-0068-1.

POZZO, María Isabel. Contenidos socioculturales sobre América Latina en manuales de español lengua extranjera de España y Argentina. Revista SURES, v.3, p.1-15. Universidad Federal de la Integración Latinoamericana. Foz de Iguazú, 2014. Disponible en: https:/ / revistas.unila. edu.br/index.php/sures/article/viewFile/146/116.

POZZO, María Isabel. Construcción de espacios interculturales en educación. Hacia el diseño e implementación de proyectos en la formación docente. En POZZO, María Isabel (ed.) Construcción de espacios interculturales en la formación docente: competencia comunicativa intercultural, cultura regional y TIC, p.17-36. Rosario: Laborde Editor, 2015a. ISBN 978-987-677-109-2.

POZZO, María Isabel. Construcción de espacios interculturales en la educación superior: competencia comunicativa intercultural, cultura regional y TIC. Foro Internacional Investigación y Políticas Públicas para la Educación. Universidad Nacional del Litoral y Ministerio de Educación de la pcia.de Santa Fe, 2015b.

POZZO, María Isabel (ed.). Construcción de espacios interculturales en la formación docente: competencia comunicativa intercultural, cultura regional y 
TIC, p. 285-306. Rosario: Laborde Editor, 2015. ISBN 978-987-677-109-2.

POZZO, María Isabel; AMEZ, Mariel; BESSO PIANETTO, María Elena et al. Diseño e implementación de proyectos interculturales como estrategia de formación de profesionales reflexivos. XII Jornada de Reflexión y Socialización del Trayecto de la Práctica: La Formación del Profesional Reflexivo. Instituto de Educación Superior N $\mathrm{N}^{\circ} 28$ “Olga Cossettini" de Rosário, 2015.

POZZO, María Isabel; BERTRAMO, Brenda; D' ASCANIO, Gabriela. La movilidad académica en la educación superior no universitaria argentina. Programas estatales y perspectivas de los actores. En POZZO María Isabel (ed.) Construcción de espacios interculturales en la formación docente: competencia comunicativa intercultural, cultura regional y TIC, p.85-98. Rosario: Laborde Editor, 2015. ISBN 978-987-677-109-2.

POZZO, María Isabel; BESSO PIANETTO, María Elena. Reflexiones históricas para el diseño de un audiovisual educativo sobre América Latina-siglo XX. Revista Culturas. Debates y perspectivas de un mundo en cambio, v.10. Facultad de Humanidades y Ciencias de la Universidad Nacional del Litoral. En prensa, 2016.

POZZO, María Isabel; BESSO PIANETTO, María Elena; DONATO, María del Carmen. Enseñar historia argentina a extranjeros a través de audiovisuales: desafíos y propuestas. En POZZO María Isabel (ed.) Construcción de espacios interculturales en la formación docente: competencia comunicativa intercultural, cultura regional 
y TIC, p.221 a 244. Rosario: Laborde Editor, 2015. ISBN 978-987-677-109-2.

POZZO,MaríaIsabel;D'ASCANIO,Gabriela;BERTRAMO, Brenda. La formación didáctica en el Profesorado de Historia del Instituto de Educación Superior $N^{\circ} 28$ Olga Cossettini. Análisis curricular. En POZZO María Isabel (ed.) Construcción de espacios interculturales en la formación docente: competencia comunicativa intercultural, cultura regional y TIC, p.131-144. Rosario: Laborde Editor, 2015. ISBN 978-987-677-109-2.

POZZO, María Isabel; JACOB, Carolina. Diversidad cultural en la escuela secundaria argentina. Perfil de los estudiantes de intercambio 2014. En POZZO María Isabel (ed.) Construcción de espacios interculturales en la formación docente: competencia comunicativa intercultural, cultura regional y TIC, p.285-306. Rosario: Laborde Editor, 2015a. ISBN 978-987-677-109-2.

POZZO María Isabel; JACOB, Carolina. Mitos y realidades sobre el uso de TIC en estudiantes de nivel superior. Perfil tecnológico de estudiantes de la carrera Historia del gran Rosario. En POZZO, María Isabel (ed.) Construcción de espacios interculturales en la formación docente: competencia comunicativa intercultural, cultura regional y TIC, p.145-154. Rosario: Laborde Editor, 2015b. ISBN 978-987-677-109-2.

POZZO, María Isabel; PIDELLO, María Amelia. La formación docente desde el enfoque por competencias. El aporte de la competencia comunicativa intercultural. En POZZO, María Isabel (ed.) Construcción de espacios 
interculturales en la formación docente: competencia comunicativa intercultural, cultura regional y TIC, p.3756. Rosario: Laborde Editor, 2015. ISBN 978-987-677-109-2.

POZZO, María Isabel; VIRGOLÍN, Natalia. La movilidad académica estudiantil como objeto de estudio. Antecedentes y estado de la cuestión. En POZZO, María Isabel (ed.) Construcción de espacios interculturales en la formación docente: competencia comunicativa intercultural, cultura regional y TIC, p.57-70. Rosario: Laborde Editor, 2015. ISBN 978-987-677-109-2.

RITACCO REAL, Maximiliano. Prólogo. En POZZO, María Isabel (ed.) Construcción de espacios interculturales en la formación docente: competencia comunicativa intercultural, cultura regional y TIC, p.9-16. Rosario: Laborde Editor, 2015. ISBN 978-987-677-109-2.

THEINER, Irene. La didactización de audiovisuales através del enfoque integrado de contenidos y lengua (EICLE). Propuestas para estudiantes de español lengua extranjera. En POZZO, María Isabel (ed.) Construcción de espacios interculturales en la formación docente: competencia comunicativa intercultural, cultura regional y TIC, p.255-272. Rosario: Laborde Editor, 2015. ISBN 978-987-677-109-2.

THEINER, Irene; POZZO, María Isabel. Competencia comunicativa intercultural y enfoque integrador de historia y lengua. Un proyecto en educación superior. Actas de las XV Jornadas y II Congreso Latinoamericano de Enseñanza de Lenguas Extranjeras en el Nivel Superior. Lenguas y Culturas: desafíos actuales de la diversidad 
y de la integración. Facultad de Lenguas, Universidad Nacional de Córdoba. En prensa, 2015.

YOUNG, Tony; SACHDEV, Itesh. Intercultural communicative competence: exploring English language beliefs and practices. Language awareness, v.20, n.2, p.81-98. 2011. 